\title{
LncRNA HOTAIR improves diabetic cardiomyopathy by increasing viability of cardiomyocytes through activation of the PI3K/Akt pathway
}

\author{
KUN QI and JIANKE ZHONG \\ Department of Gerontology, The First Hospital of Shanxi Medical University, Taiyuan, Shanxi 030001, P.R. China
}

Received March 20, 2018; Accepted July 26, 2018

DOI: $10.3892 /$ etm.2018.6755

\begin{abstract}
The current study aimed to investigate the role of long non-coding RNA (lncRNA) homeobox transcript antisense RNA (HOTAIR) in the pathogenesis of diabetic cardiomyopathy. Patients with diabetic cardiomyopathy, patients with diabetes but without cardiomyopathy and healthy controls were included in the current study. All participants underwent myocardial biopsy to collect myocardial tissues. Blood samples were also collected from each participant to prepare serum. Expression of HOTAIR in myocardial tissues was detected by reverse transcription-quantitative polymerase chain reaction. Receiver operating characteristic curve analysis was performed to evaluate the diagnostic value of serum HOTAIR for diabetic cardiomyopathy. AC16 human cardiomyocyte cells were treated with high glucose to observe the changes in expression of HOTAIR and phosphorylation of Akt. HOTAIR expression vector was transfected into cells of AC16 cell line and the effects of HOTAIR overexpression on cell viability and Akt phosphorylation were detected by MTT assay and western blot analysis, respectively. HOTAIR expression was significantly downregulated in myocardial tissues and serum of patients with diabetic cardiomyopathy compared with patients with diabetes and healthy controls. Serum HOTAIR could be used to effectively distinguish patients with diabetic cardiomyopathy from healthy controls. High glucose treatment inhibited HOTAIR expression and Akt phosphorylation. HOTAIR overexpression promoted Akt phosphorylation. HOTAIR overexpression improved AC16 cell viability, while PI3K/Akt inhibitor treatment reduced this effect. LncRNA HOTAIR may improve diabetic cardiomyopathy by increasing the viability of cardiomyocytes through activation of the PI3K/Akt pathway.
\end{abstract}

Correspondence to: Mrs. Kun Qi, Department of Gerontology, The First Hospital of Shanxi Medical University, 85 Jiefang Nan Road, Taiyuan, Shanxi 030001, P.R. China

E-mail: byqffx321kts2@163.com

Key words: diabetic cardiomyopathy, long non-coding RNA, homeobox transcript antisense RNA, phosphoinositide 3-kinase/Akt, viability, activation

\section{Introduction}

Diabetes mellitus, also known as diabetes, is a chronic disease characterized by abnormally high blood glucose levels over a prolonged period (1). With changes in lifestyle and diet, including the popularization of a western-style diet, the incidence of diabetes is increasing (2), seriously affecting patient health and lifestyle. Abnormally high glucose levels in humans may lead to multiple severe complications (3). As one of the major cardiac complications of diabetes, diabetic cardiomyopathy is also one of the leading causes of morbidity and mortality in patients with diabetes (4). At present, the treatment of diabetic cardiomyopathy is limited by the pathogenesis remaining unclear (5). Therefore, in-depth investigation of the mechanism of onset, development and progression of diabetic cardiomyopathy is required.

Studies on the pathogenesis of diabetic cardiomyopathy have revealed that its development is a complex and multi-step process with various internal and external factors involved (6). Long non-coding RNAs (lncRNAs) are a group of RNA transcripts composed of more than 200 nucleotides that lack protein-coding ability (7). The involvement of lncRNAs in cardiomyocyte viability, which is a major factor in diabetic cardiomyopathy (8), has been observed in previous studies $(9,10)$. Homeobox transcript antisense RNA (HOTAIR) is a well-studied lncRNA with critical roles in numerous human diseases, particularly in multiple types of cancer (11). A recent study reported that expression level of HOTAIR determines cardiac function in a sepsis mouse model, indicating the possible involvement of HOTAIR in heart disease (12). However, to the best of our knowledge, the involvement of HOTAIR in diabetic cardiomyopathy has not been reported. Therefore, the current study investigated the function of HOTAIR in diabetic cardiomyopathy and observed that HOTAIR improves the viability of cardiomyocytes by activating the phosphoinositide 3-kinase (PI3K)/Akt pathway. The findings of the current study may have implications for the diagnosis and treatment of diabetic cardiomyopathy.

\section{Patients and methods}

Subjects. A total of 56 patients with diabetic cardiomyopathy were recruited from March 2015 to March 2017 in The First Hospital of Shanxi Medical University (Taiyuan, China). Diagnostic criteria of diabetic cardiomyopathy were as follows: 
i) Confirmed diabetes; ii) clinical manifestations of heart failure; iii) cardiac enlargement with impaired cardiac systolic function, or diastolic dysfunction with no enlarged heart; iv) heart failure caused by heart disease such as hypertension heart disease, coronary heart disease and rheumatic valvular heart disease was excluded; v) biopsy of the heart confirmed microangiopathy and positive periodic acid-Schiff staining. Diagnostic criteria of diabetes were as follows: i) 2-h postprandial blood glucose, $\geq 11.1 \mathrm{mmol} / \mathrm{l}$; ii) fasting blood glucose, $\geq 7.0 \mathrm{mmol} / 1$; iii) HbAlc, $\geq 6.5 \%$. All patients exhibited congestive heart failure and/or arrhythmias and/or angina pectoris. Inclusion criteria were as follows: i) Patients diagnosed with diabetic cardiomyopathy and treated for the first time in The First Hospital of Shanxi Medical University; ii) patients and their families willing to participate in the study; iii) patients understood the whole experimental protocol and could cooperate with the researchers. Exclusion criteria were as follows: i) Patients with other types of cardiomyopathy; ii) patients with other diabetic complications; iii) patients treated in other hospitals prior to admission. The recruited patients included 30 males and 26 females, and the ages ranged from 37 to 72 years, with a mean age of $55.2 \pm 10.1$ years. At the same time, 44 patients with diabetes but without cardiomyopathy and 42 healthy controls were also included. The 44 patients with diabetes included 23 males and 21 females aged between 34 and 72 years, with a mean age of $53.1 \pm 8.4$ years. Patients with diabetes complicated with other diseases were not included. The 42 healthy controls included 23 males and 19 females, and the ages ranged from 36 to 69 years, with a mean age of $53.7 \pm 8.2$ years. All healthy controls exhibited normal physical conditions. No significant differences in age or sex were identified among the three groups of patients. No significant differences in body mass index (BMI) were identified between patients with diabetes and patients with diabetic cardiomyopathy, while BMI was significantly higher in patients with diabetes and patients with diabetic cardiomyopathy compared with healthy controls. The Ethics Committee of The First Hospital of Shanxi Medical University approved the study, and all patients provided written informed consent.

Specimen collection. Myocardial biopsy was performed in patients with diabetic cardiomyopathy to confirm the disease. Myocardial biopsy was also performed on all patients with diabetes and healthy controls to identify any existing heart diseases, but heart diseases were finally excluded from these patients. Whole blood $(20 \mathrm{ml})$ was obtained from each participant on the day of admission. Serum samples were prepared by keeping the blood at room temperature for $2 \mathrm{~h}$, followed by centrifugation at $1,000 \mathrm{x}$ g for $20 \mathrm{~min}$ at room temperature. Serum samples were stored in liquid nitrogen until use. Myocardial tissues were washed with ice-cold PBS and also stored in liquid nitrogen until use.

Cell line and cell culture. Human cardiomyocyte cell line AC16 was purchased from EMD Millipore (Billerica, MA, USA). AC16 cells were cultured in Dulbecco's modified Eagle's medium supplemented with $1 \%$ antibiotics (penicillin and streptomycin) and 12\% fetal bovine serum (all Sigma-Aldrich; Merck KGaA, Darmstadt, Germany) in an incubator $\left(37^{\circ} \mathrm{C}\right.$, $5 \% \mathrm{CO}_{2} / 95 \%$ air). Cells were harvested at logarithmic growth phase for subsequent experiments. To investigate the effects of high glucose on HOTAIR expression and Akt phosphorylation in human cardiomyocytes, human cardiomyocytes were treated with different concentrations of D-glucose $(20,40$ and $60 \mathrm{mM}$; Sigma-Aldrich; Merck KGaA) for $48 \mathrm{~h}$ in an incubator $\left(37^{\circ} \mathrm{C}\right.$, $5 \% \mathrm{CO}_{2} / 95 \%$ air) prior to subsequent experiments. To investigate the role of PI3K inhibition on cell viability, cells were treated with PI3K inhibitor LY294002 (10 $\mu \mathrm{M}$; Cell Signaling Technology, Inc., Danvers, MA, USA) for $24 \mathrm{~h}$ in an incubator $\left(37^{\circ} \mathrm{C}, 5 \% \mathrm{CO}_{2} / 95 \%\right.$ air $)$ prior to subsequent experiments

Cell transfection. HOTAIR cDNA (HPRM54622; GeneCopoeia, Inc., Rockville, MD, USA) was inserted into pIRSE2-EGFP vector (Clontech Laboratories, Inc., Mountainview, CA, USA) to construct the HOTAIR expression vector. Cells were cultured overnight to reach $70-80 \%$ confluence, and transfection was performed using Lipofectamine 2000 reagent (11668-019; Invitrogen; Thermo Fisher Scientific, Inc., Waltham, MA, USA) to transfect $10 \mathrm{nM}$ vector into $5 \times 10^{5}$ cells. Empty pIRSE2-EGFP vector was used as a negative control. An overexpression rate $>150 \%$ was confirmed by reverse transcription-quantitative polymerase chain reaction (RT-qPCR) prior to subsequent experiments.

MTT assay. Cell suspension with a cell density of $4 \times 10^{4}$ cells $/ \mathrm{ml}$ was prepared, and $100 \mu \mathrm{l}$ cell suspension containing $4 \times 10^{4}$ cells was added into each well of a 96-well plate. Then, $40 \mathrm{mM}$ D-glucose was added. Cells were cultured in an incubator $\left(37^{\circ} \mathrm{C}, 5 \% \mathrm{CO}_{2}\right)$ for $6 \mathrm{~h}$, then $10 \mu \mathrm{l} \mathrm{MTT}$ was added into each well. Following the addition of MTT, cells were cultured for a further $4 \mathrm{~h}$. The formazan product was dissolved in dimethyl sulfoxide. Optical density values were measured at $570 \mathrm{~nm}$.

$R T-q P C R$. Myocardial tissues were ground in liquid nitrogen, followed by the addition of TRIzol reagent (Invitrogen; Thermo Fisher Scientific, Inc.) to extract total RNA. TRIzol reagent was also mixed with serum and in vitro cultured cells to extract total RNA. A NanoDrop ${ }^{\mathrm{TM}} 2000$ spectrophotometer (Thermo Fisher Scientific, Inc.) was used to test RNA quality, and only those with a A260/A280 ratio between 1.8 and 2.0 were used to synthesize cDNA through RT using SuperScript III Reverse Transcriptase kit (Thermo Fisher Scientific, Inc.); the thermal conditions were as follows: $25^{\circ} \mathrm{C}$ for $5 \mathrm{~min}, 55^{\circ} \mathrm{C}$ for $20 \mathrm{~min}$ and $75^{\circ} \mathrm{C}$ for $15 \mathrm{~min}$. PCR reactions were performed using SYBR ${ }^{\circledR}$ Green Real-Time PCR Master Mixes (Thermo Fisher Scientific, Inc.) with the primers listed below: human HOTAIR forward, 5'-GGCGGATGCAAGTTAATAAAAC-3' and reverse, 5'-TACGCCTGAGTGTTCACGAG-3'; human $\beta$-actin forward, 5'-GACCTCTATGCCAACACAGT-3' and reverse, 5'-AGTACTTGCGCTCAGGAGGA-3'. PCR reaction conditions were as follows: $95^{\circ} \mathrm{C}$ for $30 \mathrm{sec}$, followed by 40 cycles of $95^{\circ} \mathrm{C}$ for $20 \mathrm{sec}$ and $60^{\circ} \mathrm{C}$ for $35 \mathrm{sec}$. Data were processed using the $2^{-\Delta \Delta \mathrm{Cq}}$ method (13), and expression of HOTAIR was normalized to endogenous control $\beta$-actin.

Western blot analysis. Radioimmunoprecipitation assay buffer (Cell Signaling Technology, Inc.) was mixed with in vitro cultured cells to extract total protein, followed by quantification using the BCA method. Electrophoresis was performed using $10 \%$ SDS-PAGE with $20 \mu \mathrm{g}$ protein from each sample. Proteins 

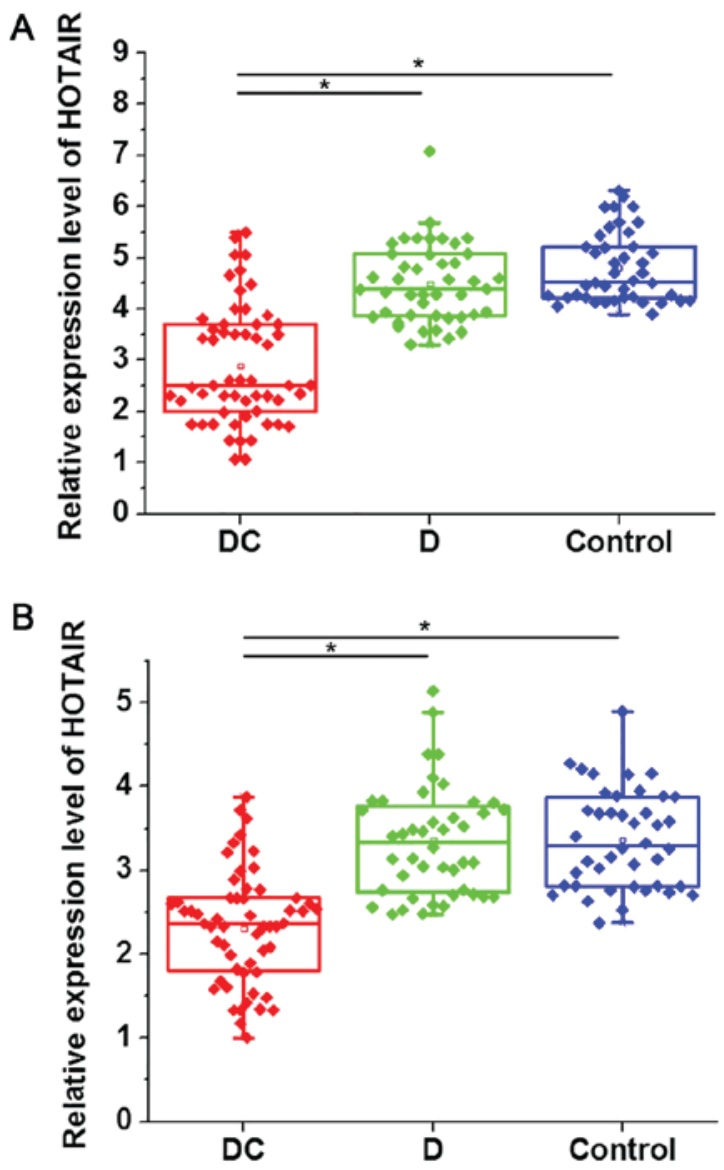

Figure 1. Expression of HOTAIR in different groups of participants. The figure indicates HOTAIR expression in (A) myocardial tissues and (B) serum of different groups of participants. DC, diabetic cardiomyopathy; D, diabetes without cardiomyopathy; HOTAIR, homeobox transcript antisense RNA. ${ }^{*} \mathrm{P}<0.05$.

were transferred to a PVDF membrane, followed by blocking with $5 \%$ skimmed milk at room temperature for $2 \mathrm{~h}$. Following washing, membranes were incubated with primary antibodies, including rabbit anti-Akt antibody (cat. no. ab126811), anti-p-Akt (phospho T308; cat. no. ab38449) and anti-GAPDH (cat. no. ab8245; all 1:2,000; Abcam), overnight at $4^{\circ} \mathrm{C}$. Following washing, anti-rabbit IgG-horseradish peroxidase secondary antibody (1:1,000; MBS435036; MyBioSource, Inc., San Diego, CA, USA) was incubated with the membranes at room temperature for $1 \mathrm{~h}$. Following washing, enhanced chemiluminescence (Sigma-Aldrich; Merck KGaA) was used to detect signals. Image J v1.6 software (National Institutes of Health, Bethesda, MD, USA) was used to normalize relative expression of each protein to endogenous control GAPDH.

Statistical analysis. SPSS 19.0 (IBM Corp., Armonk, NY, USA) was used to perform statistical analysis. All experiments were performed in triplicate. Categorical data were compared by chi-square test. Continuous data are presented as mean \pm standard deviation and were compared among multiple groups by one-way analysis of variance followed by least significant difference post-hoc test. Receiver operating characteristic (ROC) curve analysis was performed to evaluate the diagnostic value of expression level of HOTAIR in myocardial tissues (cutoff value, 2.33) and
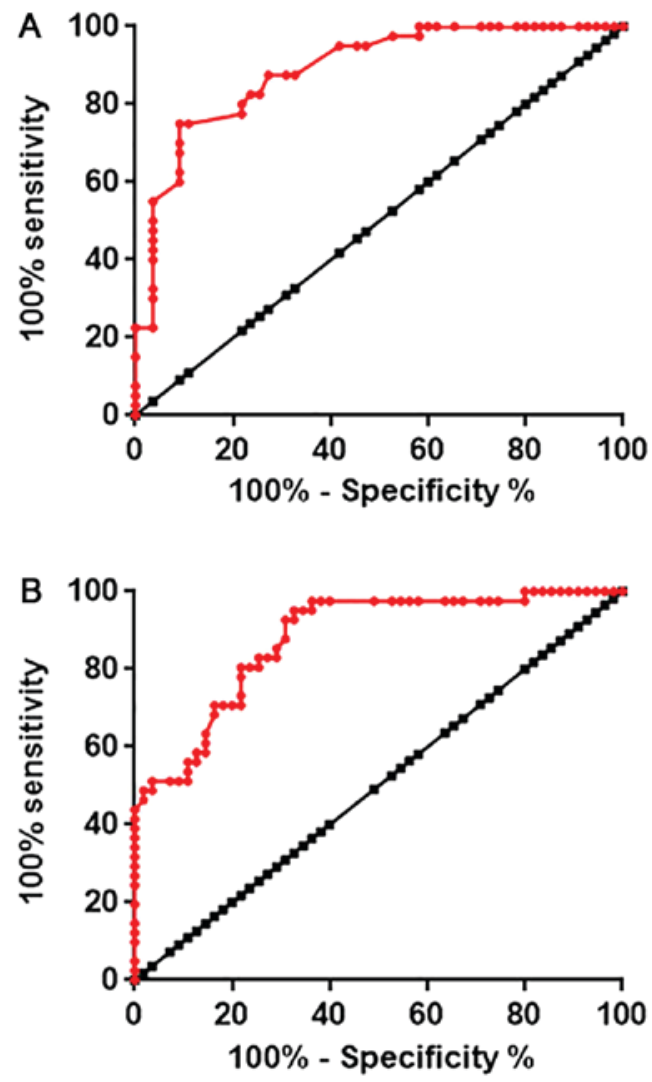

Figure 2. Diagnostic values of HOTAIR expression for diabetic cardiomyopathy. This figure indicates the receiver operating characteristic curve for the diagnosis of diabetic cardiomyopathy using HOTAIR expression in (A) myocardial tissues and (B) serum. HOTAIR, homeobox transcript antisense RNA.

serum (cutoff value, 2.45) for diabetic cardiomyopathy. For the analysis of the association between expression level of HOTAIR and clinicopathological data of patients with diabetic cardiomyopathy, patients with smoking habits were defined as patients who smoked $>3$ cigarettes per day and $>3$ days per week, and patients with drinking habits were defined as patient who drank $>2$ times per week. $\mathrm{P}<0.05$ was considered to indicate a statistically significant difference.

\section{Results}

Expression of HOTAIR in myocardial tissues and serum is specifically downregulated in patients with diabetic cardiomyopathy. RT-qPCR was performed to detect the expression of HOTAIR in myocardial tissues and serum of all participants. As indicated in Fig. 1, the expression level of HOTAIR in myocardial tissues (Fig. 1A) and serum (Fig. 1B) was significantly downregulated in patients with diabetic cardiomyopathy compared with patients with diabetes and healthy controls. However, no significant differences in HOTAIR expression were identified between diabetic patients without cardiomyopathy and healthy controls.

Diagnostic values of HOTAIR expression for diabetic cardiomyopathy. Receiver operating characteristic (ROC) curve analysis was performed to evaluate the diagnostic value of expression level of HOTAIR in myocardial tissues and serum for diabetic cardiomyopathy. As indicated in Fig. 2A, 
Table I. Associations between expression level of HOTAIR in myocardial tissues and clinicopathological data of patients with diabetic cardiomyopathy.

\begin{tabular}{|c|c|c|c|c|c|c|}
\hline Characteristic & Group & Cases & $\begin{array}{c}\text { High expression of } \\
\text { HOTAIR }\end{array}$ & $\begin{array}{l}\text { Low expression } \\
\text { of HOTAIR }\end{array}$ & $\chi^{2}$ & P-value \\
\hline \multirow[t]{2}{*}{ Sex } & Male & 30 & 17 & 13 & \multirow[t]{2}{*}{0.63} & \multirow[t]{2}{*}{0.43} \\
\hline & Female & 26 & 11 & 15 & & \\
\hline \multirow[t]{2}{*}{ Age (years) } & $>50$ & 29 & 16 & 13 & \multirow[t]{2}{*}{0.64} & \multirow[t]{2}{*}{0.42} \\
\hline & $<50$ & 27 & 12 & 15 & & \\
\hline \multirow[t]{2}{*}{ Course of disease (years) } & $>2$ & 33 & 12 & 21 & \multirow[t]{2}{*}{5.98} & \multirow[t]{2}{*}{0.02} \\
\hline & $<2$ & 23 & 16 & 7 & & \\
\hline \multirow[t]{2}{*}{ Smoking } & Yes & 25 & 14 & 11 & \multirow[t]{2}{*}{0.65} & \multirow[t]{2}{*}{0.42} \\
\hline & No & 31 & 14 & 17 & & \\
\hline \multirow[t]{2}{*}{ Drinking } & Yes & 28 & 12 & 16 & \multirow[t]{2}{*}{1.14} & \multirow[t]{2}{*}{0.29} \\
\hline & No & 28 & 16 & 12 & & \\
\hline
\end{tabular}

HOTAIR, homeobox transcript antisense RNA.

Table II. Associations between expression level of HOTAIR in serum and clinicopathological data of patients with diabetic cardiomyopathy.

\begin{tabular}{|c|c|c|c|c|c|c|}
\hline Characteristic & Group & Cases & $\begin{array}{l}\text { High expression } \\
\text { of HOTAIR }\end{array}$ & $\begin{array}{c}\text { Low expression } \\
\text { of HOTAIR }\end{array}$ & $\chi^{2}$ & P-value \\
\hline \multirow[t]{2}{*}{ Sex } & Male & 30 & 16 & 14 & \multirow[t]{2}{*}{0.29} & \multirow[t]{2}{*}{0.59} \\
\hline & Female & 26 & 12 & 14 & & \\
\hline \multirow[t]{2}{*}{ Age (years) } & $>50$ & 29 & 17 & 12 & \multirow[t]{2}{*}{1.79} & \multirow[t]{2}{*}{0.18} \\
\hline & $<50$ & 27 & 11 & 16 & & \\
\hline \multirow[t]{2}{*}{ Course of disease (years) } & $>5$ & 33 & 12 & 21 & \multirow[t]{2}{*}{5.98} & \multirow[t]{2}{*}{0.02} \\
\hline & $<5$ & 23 & 16 & 7 & & \\
\hline \multirow[t]{2}{*}{ Smoking } & Yes & 25 & 14 & 11 & \multirow[t]{2}{*}{1.64} & \multirow[t]{2}{*}{0.2} \\
\hline & No & 31 & 14 & 17 & & \\
\hline \multirow[t]{2}{*}{ Drinking } & Yes & 28 & 11 & 17 & \multirow[t]{2}{*}{2.57} & \multirow[t]{2}{*}{0.11} \\
\hline & No & 28 & 17 & 11 & & \\
\hline
\end{tabular}

HOTAIR, homeobox transcript antisense RNA.

the area under the curve (AUC) for the use of HOTAIR expression in myocardial tissues was 0.8880 , with $95 \%$ confidence interval of $0.8231-0.9526$ and standard error of 0.03309 $(\mathrm{P}<0.0001)$. In addition, AUC for the use of serum HOTAIR in the diagnosis of diabetic cardiomyopathy was 0.8780 , with 95\% confidence interval of 0.8107-0.9454 and standard error of 0.03436 ( $\mathrm{P}<0.0001$; Fig. 2B). Those data suggested that HOTAIR expression may serve as an effective diagnostic marker for diabetic cardiomyopathy.

Association between expression level of HOTAIR and clinicopathological data of patients with diabetic cardiomyopathy. Patients were divided into a high expression group and a low expression group according to the median expression level of HOTAIR in myocardial tissues and serum. A chi-square test was performed to analyze the association between expression of HOTAIR and the clinicopathological data of patients with diabetic cardiomyopathy. As indicated in Tables I and II, expression of HOTAIR in myocardial tissues (Table I) and serum (Table II) of patients with diabetic cardiomyopathy exhibited no significant associations with age, sex or smoking and drinking habits, but was significantly associated with the disease course $(\mathrm{P}<0.05)$.

Effects of high glucose on HOTAIR expression and Akt phosphorylation in human cardiomyocytes. Human cardiomyocytes were treated with different concentrations of D-glucose (20, 40 and $60 \mathrm{mM}$ ) for $48 \mathrm{~h}$, and the expression of HOTAIR and Akt was detected by RT-qPCR and western blot analysis, respectively. As indicated in Fig. 3A, D-glucose treatment decreased the expression level of HOTAIR and expression was reduced with higher doses of D-glucose in 

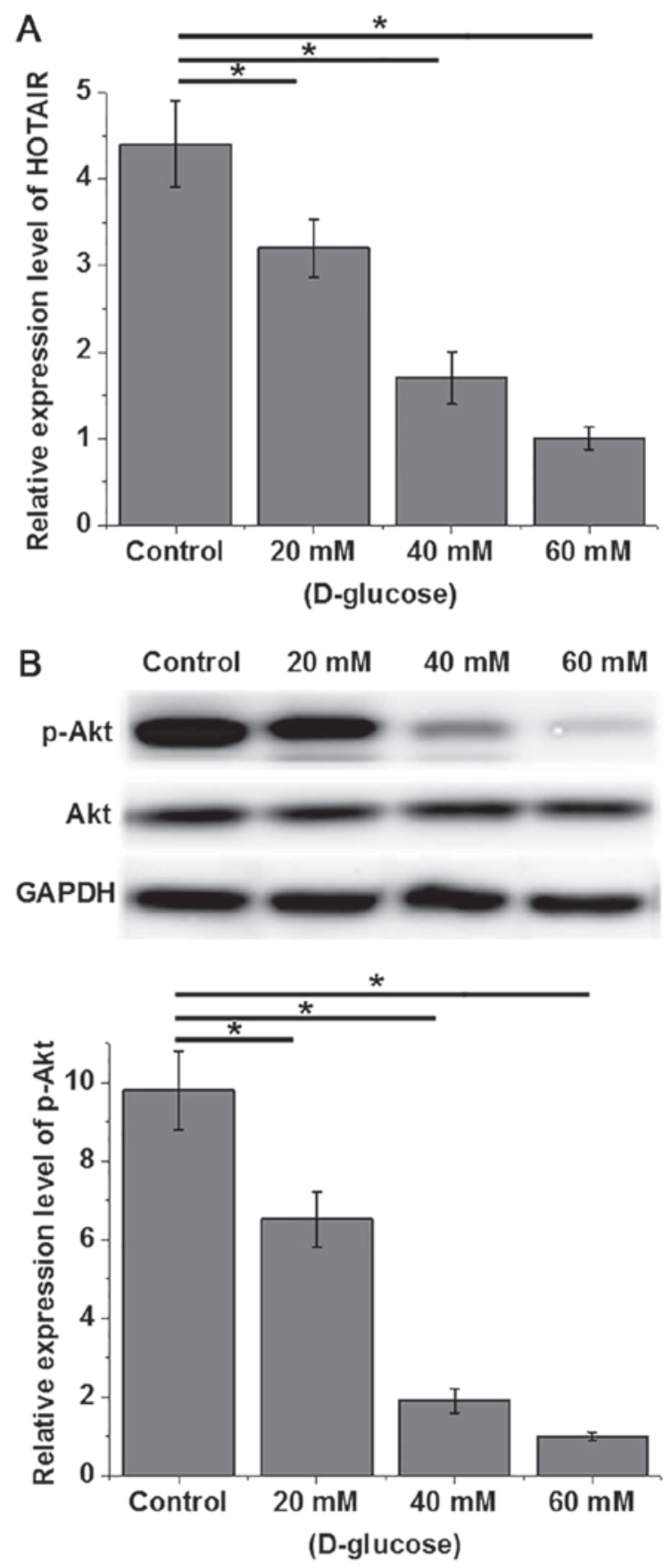

Figure 3. Effects of high glucose on HOTAIR expression and Akt phosphorylation in human cardiomyocytes. The figure indicates the effects of high glucose on (A) HOTAIR expression and (B) Akt phosphorylation in human cardiomyocytes. ${ }^{*} \mathrm{P}<0.05$. HOTAIR, homeobox transcript antisense RNA; p-, phosphorylated.

what appeared to be a dose dependent manner $(\mathrm{P}<0.05)$. Although D-glucose exhibited no significant effects on Akt protein expression, phosphorylation levels of Akt decreased with increasing concentrations of D-glucose (Fig. 3B; $\mathrm{P}<0.05)$. Therefore, a high glucose environment can lead to the downregulation of HOTAIR expression and decreased phosphorylation of Akt.

Effects of HOTAIR overexpression and Akt phosphorylation in human cardiomyocytes. An AC16 cell line with HOTAIR overexpression was constructed and confirmed by RT-qPCR

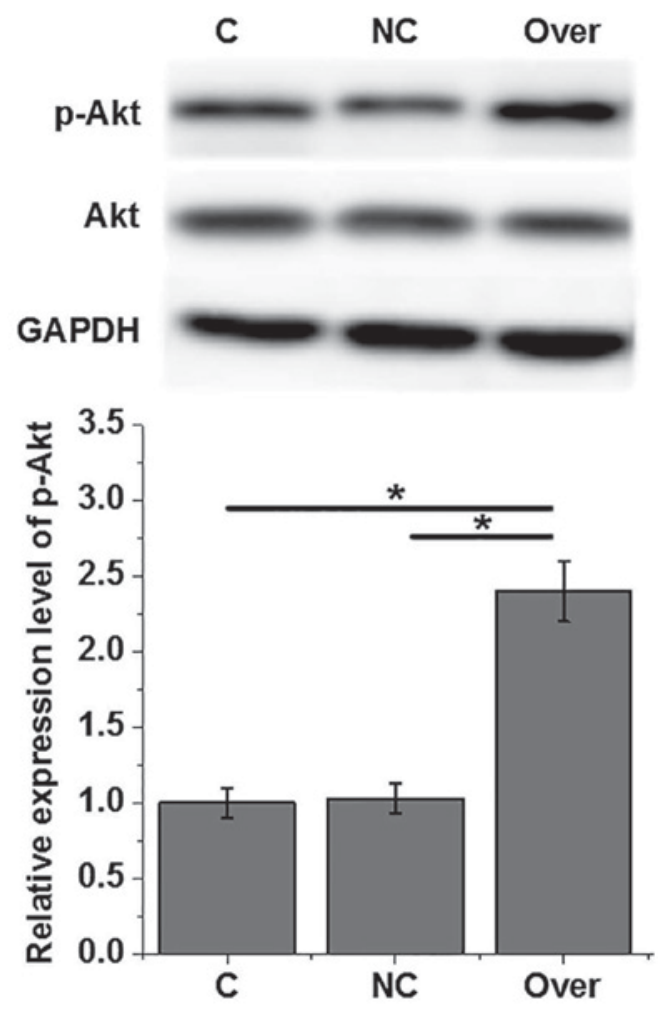

Figure 4. Effect of HOTAIR overexpression on Akt phosphorylation in human cardiomyocytes. ${ }^{*} \mathrm{P}<0.05$. C, control (non-transfected); $\mathrm{NC}$, negative control (empty vector); Over, HOTAIR overexpression (transfected with overexpression vector); HOTAIR, homeobox transcript antisense RNA; p-, phosphorylated.

(data not shown). As indicated in Fig. 4, HOTAIR overexpression exerted no significant effects on Akt expression, but significantly increased the phosphorylation level of Akt $(\mathrm{P}<0.05)$. Therefore, HOTAIR overexpression can upregulate the phosphorylation of Akt in human cardiomyocytes.

Effects of HOTAIR overexpression on the viability of AC16 cells under high glucose treatment. AC16 cells were treated with $40 \mathrm{mM}$ D-glucose and cell viability was measured by MTT assay. As indicated in Fig. 5, compared with control cells without transfection and negative control cells transfected with empty vector, HOTAIR overexpression significantly increased cell viability $(\mathrm{P}<0.05)$. However, treatment with PI3K inhibitor LY294002 $(10 \mu \mathrm{M})$ significantly reduced the effect of HOTAIR overexpression on cell viability $(\mathrm{P}<0.05)$. Therefore, HOTAIR may increase the viability of AC16 cells by activating the PI3K signaling pathway.

\section{Discussion}

High blood glucose levels in diabetic patients have significant effects on the expression of a large set of lncRNAs, and altered expression of IncRNAs may participate in the pathogenesis of diabetes-associated complications that promote or inhibit disease progression (14). The development of diabetic cardiomyopathy, which is a major complication in diabetes, is usually accompanied by changes in expression patterns of certain lncRNAs. It has been reported that IncRNA myocardial infarction-associated transcript 
(MIAT) expression is upregulated in patients with diabetic cardiomyopathy compared with healthy controls, and upregulated lncRNA MIAT expression reverses the inhibitory effect of microRNA-22-3p to promote the development of disease (15). In another study, downregulation of lncRNA metastasis-associated lung adenocarcinoma transcript-1 was indicated to improve cardiomyocyte viability, which in turn inhibited the occurrence of diabetic cardiomyopathy (16). HOTAIR is a well-studied lncRNA with altered expression in numerous human diseases, particularly in multiple types of cancer (11). However, the expression pattern of HOTAIR in diabetes and diabetes-associated complications has not been well characterized. In the current study, HOTAIR expression was identified to be upregulated in patients with diabetic cardiomyopathy but not in patients with diabetes without cardiomyopathy. These data suggested that HOTAIR may specifically participate in the development of cardiomyopathy in patients with diabetes.

Diabetic cardiomyopathy is frequently undiagnosed, and delayed treatment usually leads to heart failure, which has a high mortality rate (17). Therefore, early diagnosis and treatment is critical for the survival of patients. In the current study, ROC analysis identified that HOTAIR expression in both myocardial tissues and serum could be used to accurately distinguish patients with diabetic cardiomyopathy from healthy controls, indicating that HOTAIR may serve as a diagnostic marker for diabetic cardiomyopathy. In addition, detection of serum HOTAIR through blood sampling as a less invasive procedure may be preferred over myocardial biopsy.

Expression of lncRNAs is affected by numerous factors, including aging (18), alcohol abuse (19) and tobacco consumption (20). In the current study, expression of HOTAIR exhibited no significant associations with patient age or smoking and drinking habits. These data suggest that HOTAIR may serve as an effective and reliable biomarker for diabetic cardiomyopathy.

In the current study, treatment with high levels of glucose significantly reduced the expression level of HOTAIR in human cardiomyocytes and expression was reduced at higher doses of D-glucose in what appeared to be a dose dependent manner. High levels of glucose also inhibited the phosphorylation of Akt. It has been reported previously that activation of the PI3K/Akt pathway may improve viability of cardiomyocytes under ischemia conditions (21), and reduced viability and increased apoptosis of cardiomyocytes are the main pathological changes in diabetic cardiomyopathy (22). In addition, it is well established that HOTAIR exerts its biological effects via interactions with the PI3K/Akt pathway (23). In the current study, HOTAIR overexpression significantly promoted the phosphorylation of Akt, indicating that there was cross-talk between HOTAIR and the PI3K/Akt pathway in cardiomyocytes. It is known that HOTAIR is involved in the regulation of viability of multiple cell types $(24,25)$. In the current study, HOTAIR overexpression significantly improved viability of cardiomyocytes, while treatment with PI3K inhibitor significantly reduced this effect. However, treatment with PI3K inhibitor exhibited no significant effects on HOTAIR expression (data not shown). These data suggest that HOTAIR may be involved in diabetic

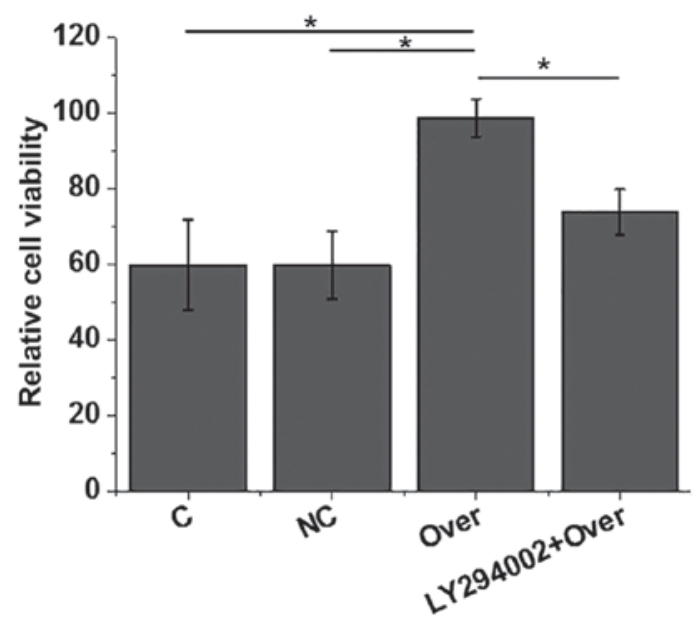

Figure 5. Effect of HOTAIR expression on cell viability. ${ }^{*} \mathrm{P}<0.05$. C, control (non-transfected); NC, negative control (empty vector); Over, HOTAIR overexpression (transfected with overexpression vector); HOTAIR, homeobox transcript antisense RNA.

cardiomyopathy by improving viability of cardiomyocytes via activation of the PI3K/Akt pathway.

It worth noting that although high levels of glucose suppressed the expression of HOTAIR in human cardiomyocytes, patients with diabetes without cardiomyopathy, who would be expected to have high plasma glucose levels, did not exhibit significantly downregulated HOTAIR expression compared with healthy controls. A possible explanation for this is that both heart lesions and high glucose downregulated HOTAIR, while high blood glucose itself in patients with diabetes without cardiomyopathy does not induce significant changes. In addition, in vitro experiments may not fully mimic in vivo conditions. Further investigations are required in this area.

In conclusion, HOTAIR expression was specifically downregulated in patients with diabetic cardiomyopathy. Serum HOTAIR may serve as a promising biomarker for diabetic cardiomyopathy. High glucose treatment inhibited HOTAIR expression and Akt phosphorylation. HOTAIR overexpression promoted Akt phosphorylation and improved AC16 cell viability. PI3K/Akt inhibitor treatment reduced this enhancing effect of HOTAIR overexpression on AC16 cell viability. These results suggest that lncRNA HOTAIR may improve diabetic cardiomyopathy by improving the viability of cardiomyocytes through activation of the PI3K/Akt pathway.

\section{Acknowledgements}

Not applicable.

\section{Funding}

No funding was received.

\section{Availability of data and materials}

All data generated or analyzed during this study are included in this published article. 


\section{Authors' contributions}

$\mathrm{KQ}$ and $\mathrm{JZ}$ were responsible for the conception and design of the study, and revised the manuscript. KQ and JZ also performed the experiments. KQ analyzed and interpreted the data, and drafted the manuscript.

\section{Ethics approval and consent to participate}

The Ethics Committee of The First Hospital of Shanxi Medical University approved the study and all patients provided written informed consent.

\section{Patient consent for publication}

Not applicable.

\section{Competing interests}

The authors declare that they have no competing interest.

\section{References}

1. Alberti KG and Zimmet PZ: Definition, diagnosis and classification of diabetes mellitus and its complications. Part 1: Diagnosis and classification of diabetes mellitus. Provisional report of a WHO Consultation. Diabetic Med 15: 539-553, 1998.

2. Imamura F, O'Connor L, Ye Z, Mursu J, Hayashino Y, Bhupathiraju SN and Forouhi NG: Consumption of sugar sweetened beverages, artificially sweetened beverages, and fruit juice and incidence of type 2 diabetes: Systematic review, meta-analysis, and estimation of population attributable fraction. BMJ 351: h3576, 2015.

3. Nathan DM: DCCT/EDIC Research Group: The diabetes control and complications trial/epidemiology of diabetes interventions and complications study at 30 years: Overview. Diabetes Care 37: 9-16, 2014

4. Bugger H and Abel ED: Molecular mechanisms of diabetic cardiomyopathy. Diabetologia 57: 660-671, 2014.

5. Huynh K, Bernardo BC, McMullen JR and Ritchie RH: Diabetic cardiomyopathy: Mechanisms and new treatment strategies targeting antioxidant signaling pathways. Pharmacol Ther 142: $375-415,2014$

6. Jia G, DeMarco VG and Sowers JR: Insulin resistance and hyperinsulinaemia in diabetic cardiomyopathy. Nat Rev Endocrinol 12: 144-153, 2016.

7. Fatica A and Bozzoni I: Long non-coding RNAs: New players in cell differentiation and development. Nat Rev Gen 15: 7-21, 2014

8. Nunes S, Rolo AP, Palmeira CM and Reis F: Diabetic cardiomyopathy: Focus on oxidative stress, mitochondrial dysfunction and inflammation. Cardiomyopathies-Types and Treatments. InTech, 2017.
9. Li X, Wang H, Yao B, Xu W, Chen J and Zhou X: lncRNA H19/miR-675 axis regulates cardiomyocyte apoptosis by targeting VDAC1 in diabetic cardiomyopathy. Sci Rep 6: 36340 , 2016.

10. Wang K, Long B, Zhou LY, Liu F, Zhou QY, Liu CY, Fan YY and Li PF: CARL lncRNA inhibits anoxia-induced mitochondrial fission and apoptosis in cardiomyocytes by impairing miR-539-dependent PHB2 downregulation. Nat Commun 5: 3596, 2014.

11. Gupta RA, Shah N, Wang KC, Kim J, Horlings HM, Wong DJ, Tsai MC, Hung T, Argani P, Rinn JL, et al: Long non-coding RNA HOTAIR reprograms chromatin state to promote cancer metastasis. Nature 464: 1071-1076, 2010.

12. Wu H, Liu J, Li W, Liu G and Li Z: LncRNA-HOTAIR promotes TNF- $\alpha$ production in cardiomyocytes of LPS-induced sepsis mice by activating NF- $\kappa \mathrm{B}$ pathway. Biochem Biophys Res Commun 471: 240-246, 2016.

13. Livak KJ and Schmittgen TD: Analysis of relative gene expression data using real-time quantitative PCR and the 2(-Delta Delta C(T)) method. Methods 25: 402-408, 2001.

14. Leung A, Amaram V and Natarajan R: Linking diabetic vascular complications with LncRNAs. Vasc Pharmacol, 2018.

15. Zhou X, Zhang W, Jin M, Chen J, Xu W and Kong X: lncRNA MIAT functions as a competing endogenous RNA to upregulate DAPK 2 by sponging miR-22-3p in diabetic cardiomyopathy. Cell death Dis 8: 2929, 2017.

16. Zhang M, Gu H, Xu W and Zhou X: Down-regulation of lncRNA MALAT1 reduces cardiomyocyte apoptosis and improves left ventricular function in diabetic rats. Int J cardiol 203: 214-216, 2016.

17. Marcinkiewicz A, Ostrowski S and Drzewoski J: Can the onset of heart failure be delayed by treating diabetic cardiomyopathy? Diabetol Metab Syndr 9: 21, 2017.

18. Grammatikakis I, Panda AC, Abdelmohsen K and Gorospe M: Long noncoding RNAs (lncRNAs) and the molecular hallmarks of aging. Aging (Albany NY) 6: 992, 2014.

19. Mayfield RD: Emerging roles for ncRNAs in alcohol use disorders. Alcohol 60: 31-39, 2017

20. Wang J, Qiu M, Xu Y, Li M, Dong G, Mao Q, Yin R and Xu L: Long noncoding RNA CCAT2 correlates with smoking in esophageal squamous cell carcinoma. Tumor Biol 36: 5523-5528, 2015.

21. Chen S, Liu J, Liu X, Fu Y, Zhang M, Lin Q, Zhu J, Mai L, Shan $\mathrm{Z}, \mathrm{Yu} \mathrm{X}$, et al: Panax notoginseng saponins inhibit ischemia-induced apoptosis by activating PI3K/Akt pathway in cardiomyocytes. J Ethnopharmacol 137: 263-270, 2011.

22. Cai L and Kang YJ: Cell death and diabetic cardiomyopathy. Cardiovasc Toxicol 3: 219-228, 2003.

23. Yan J, Dang Y, Liu S, Zhang Y and Zhang G: LncRNA HOTAIR promotes cisplatin resistance in gastric cancer by targeting miR-126 to activate the PI3K/AKT/MRP1 genes. Tumor Biol, 2016.

24. Qiu JJ, Wang Y, Ding JX, Jin HY, Yang G and Hua KQ: The long non-coding RNA HOTAIR promotes the proliferation of serous ovarian cancer cells through the regulation of cell cycle arrest and apoptosis. Exp Cell Res 333: 238-248, 2015.

25. Chen J, Lin C, Yong W, Ye Y and Huang Z: Calycosin and genistein induce apoptosis by inactivation of HOTAIR/p-Akt signaling pathway in human breast cancer MCF-7 cells. Cell Physiol Biochem 35: 722-728, 2015. 